Check for updates

Cite this: RSC Adv., 2018, 8, 36257

\title{
Engineering the interface in mechanically responsive graphene-based films $\uparrow$
}

\author{
Yaqing Chen, ${ }^{\text {ab }}$ Zhaohe Dai, (D) a Chuanxin Weng, ${ }^{\text {ab }}$ Guorui Wang, ${ }^{a}$ Xuelu Liu, ${ }^{c}$ \\ Xin Cong, ${ }^{c}$ Pingheng Tan, (D) ${ }^{c}$ Luqi Liu (D) ${ }^{* a}$ and Zhong Zhang (D)*a
}

Due to their extraordinary mechanical properties, nanocarbon materials (e.g. carbon nanotube and graphene) are attracting great interests in the field of nanocomposites. One unique feature in nanocarbon-based nanocomposites is their intrinsically rich interface, allowing them to adapt the microstructures in response to external loading and, in turn, to stiffen themselves. This mechanical behavior, called responsive stiffening, was usually observed in biological materials such as bones and muscles. The mechanically responsive behaviors of nanocarbon-based materials are particularly exciting because the nanocarbon-enabled huge interface area offers opportunities to tune such stiffening performance while this interface advantage is not fully exploited yet. Here, we demonstrate stiffening behaviors in graphene oxide (GO)-based film materials in response to dynamic oscillations. Through a facile method of polymer content alteration and alkali treatment, the microstructure and interlayer interaction of GO films are modified, along with the resulted responsively stiffening performance. Based on polarized Raman spectra characterizations, we attribute the stiffening mechanism to the microstructural evolution of GO films during dynamic tension as well as the polymer chains alignment. Finally, we highlight the significantly improved static mechanical properties of GO film after a simple stiffening process. Our results not only aid in the development of biomimetic, adaptive materials, but provide a mechanical way for the design of high-performance nanocarbon-based nanocomposites.

Received 26th September 2018
Accepted 20th October 2018

DOI: 10.1039/c8ra07974a

rsc.li/rsc-advances dynamic load, even though such load is far lower than the yielding strength of this material., ${ }^{7,8}$ In efforts to achieve outstanding performance and maintain the mechanical reliability in practical applications, the bioinspired mechanically responsive behaviors may be exploited to improve the resiliency of manmade materials to repeated loading.

Recently, there is increasing evidence in the literature for biomimetic behaviors (which includes self-stiffening) observed in various nanocarbon-based macroscopic materials. ${ }^{9-13}$ Carey et al. found the dynamic-stress-induced stiffening in polydimethylsiloxane (PDMS)/carbon nanotube (CNT) nanocomposite, ${ }^{11}$ and Cao et al. observed that PDMS/graphene oxide (GO) also exhibited strain hardening under dynamic compression. ${ }^{9}$ Stiffening effects under cyclic tension were also observed in nanocomposite system such as polyacrylonitrile/CNT demonstrated by Li et al., however, it was reported that polyacrylonitrile/GO showed no obvious stiffening behavior. ${ }^{13}$ Furthermore, Dai et al. reported the stiffening behavior of GObased films with layered structure, but tuning of stiffening degree is limited and can only be affected by interlayer adhesion of GO sheets. ${ }^{10}$ The stiffening mechanism in these nanocarbonbased nanocomposites system was attributed to interfacial structural evolution, where the ultrahigh aspect ratios of nanocarbons intrinsically feature huge interface area. More broadly, except for the nanocarbon systems, stiffening
${ }^{a}$ CAS Key Laboratory of Nanosystem and Hierarchical Fabrication, CAS Center for Excellence in Nanoscience, National Center for Nanoscience and Technology, Beijing, 100190, People's Republic of China. E-mail: liulq@nanoctr.cn; zhong. zhang@nanoctr.cn

${ }^{b}$ University of Chinese Academy of Science, Beijing 100049, People's Republic of China ${ }^{c}$ State Key Laboratory of Superlattices and Microstructures, Chinese Academy of Sciences, Beijing 100083, People's Republic of China

$\dagger$ Electronic supplementary information (ESI) available. See DOI: $10.1039 / \mathrm{c} 8 \mathrm{ra07974a}$ 
behaviors are observed in materials where the microstructures are able to remodel and reorient in response to loadings. For example, Senses and Akcora observed an interface-driven stiffening under oscillatory shear in poly(methyl methacrylate)/ nanosilica particle nanocomposite. ${ }^{14}$ Agrawal et al. observed the dynamic self-stiffening in liquid crystal elastomers attributed to a mobile nematic director rotating under dynamic compression. ${ }^{15}$ However, the development of mechanically responsive materials is in its infancy, and further tuning the self-stiffening performance is still elusive.

Here, we report that GO-based nanocomposite films exhibit the analogous stiffening behavior when subjected to repeated tensile loads. The GO-based nanocomposite films are constructed with $\mathrm{GO}^{\mathbf{1 6 , 1 7}}$ nanosheets (rich in hydroxylic, carboxylic and epoxy groups) as "brick" and chitosan ${ }^{18}$ (CS, rich in hydroxylic and amine groups) as "mortar" through covalent and hydrogen bonding in the interface. Such structural characteristic enables individual sheets to remodel and realign in response to external dynamic load, leading to obvious stiffening behavior of the bulk material. Through altering their microstructures and interfaces, i.e. interlayer spacing by the content of intercalated macromolecules and the interlayer interaction by chemical treatment, we effectively modulated the stiffening behaviors of GO films. According to our polarized Raman spectra characterizations we found that the major stiffening behavior is a process of layered structural evolutions under repeated loading-that the external energy gives rise to the regularity of layered structure and the better stress transfer across the interface.

\section{Experimental section}

\subsection{Material}

Graphite was obtained from Qingdao Yingshida Graphite Co., Ltd., China. Graphene oxide was synthesized by a modified Hummer's method from the purified natural graphite. Longchain chitosan (degree of deacetylation: ca. 90\%, viscosity: $1012 \mathrm{mPa}$ s for $1 \%(\mathrm{w} / \mathrm{v})$, molecular weight: $c a .300 \mathrm{kDa})$ was purchased from Shandong Jinhu Co., Ltd., China.

\subsection{Preparation of GO/CS nanocomposite films and as prepared GO films}

GO sheets were dispersed in deionized water $\left(0.5 \mathrm{mg} \mathrm{mL}^{-1}\right)$ and further exfoliated via ultrasonic agitation $(100 \mathrm{~W})$ for 1 hour to ensure the well dispersion. Chitosan (CS) flakes (1 wt\%) was dissolved in an aqueous acidic solution of glacial acetic acid ( $0.5 \mathrm{wt} \%$ ) for 24 hours. The GO dispersion was then gradually added into the CS solution with intensely stirring and drops of alkali solution ${ }^{19,20}$ to yield a uniform brown-colored dispersion for 24 hours when GO nanosheets were enwrapped by CS chains. By means of vacuum-assisted filtration method, these hybrid building blocks were filtrated by a cellulose membrane filter $(0.22 \mu \mathrm{m}$ pore size) and assembled into GO/CS nanocomposite films, following air drying and peeling off from the filter. A series of GO/CS nanocomposites with different weight ratios $(100 / 0,93 / 7,78 / 22,68 / 32)$ were prepared. All the films were dried in the drying oven at $60{ }^{\circ} \mathrm{C}$ for 12 hours before test.

\subsection{Preparation of $\mathrm{GO} / \mathrm{CS}-\mathrm{NaOH}$ nanocomposite films and GO-NaOH films}

The as-prepared GO/CS thin films were immersed in $2 \mathrm{M}$ of aqueous $\mathrm{NaOH}$ for 24 hours and were fully rinsed and immersed in deionized water for 12 hours. They were then allowed to be dried at $60{ }^{\circ} \mathrm{C}$ for 12 hours in an drying oven.

\subsection{Characterizations}

The cross sections of specimen were observed by field-emission scanning electron microscopy (SEM, JSM-7500 F) under voltage of $5 \mathrm{kV}$. X-ray diffraction (XRD) measurements for films were studied at room temperature ( $\mathrm{Cu} \mathrm{K} \alpha$ radiation, D/MAX-TTR III, CBO, Japan) with a voltage of $40.0 \mathrm{kV}$, current of $200.0 \mathrm{~mA}$, and scanning speed of 10.0 degrees per minute. Thermogravimetry analysis (TGA) was performed in TA (Q500, Waters LLC in USA) with a heating rate of $10{ }^{\circ} \mathrm{C} \mathrm{min}^{-1}$ under nitrogen from 30 to $800{ }^{\circ} \mathrm{C}$. Spectral analysis was conducted by X-ray photoelectron spectroscopy (XPS) with an ESCALab220i-XL electron spectrometer from VG Scientific using $300 \mathrm{~W}$ Al K $\alpha$ radiation. A specimen teared by tape along base plane of the GO-based films was irradiated with an X-ray beam. The XPS measurements were performed before and after each film treatment. Polarized Raman spectra were analyzed to study the orientation of GO/CS nanosheets before and after dynamic loading. Jobin-Yvon HR800 micro-Raman system (with an excitation wavelength of $633 \mathrm{~nm}$ from a He-Ne laser) was utilized equipped with an $\times 100$ objective lens (numerical aperture $\approx 0.90$ ), a liquidnitrogen-cooled charge couple detector, and a 600 lines per $\mathrm{mm}$ grating. A rotational stage and a motorized $\mathrm{x}-\mathrm{y}$ stage was used to vary the angle of films in respect to the vector of the linearly polarized excitation. All featured bands in Raman spectra of graphene were fitted with Lorentzian functions to obtain the Raman shifts.

\subsection{Mechanical testing}

Mechanical performances of GO-based films were measured by a dynamic mechanical analyzer (DMA, TA Q800, Waters LLC in USA) isothermally at room temperature $\left(25^{\circ} \mathrm{C}\right)$. Samples of prepared GO-based films with thickness of $\sim 5 \mu \mathrm{m}$ were cut by a razor blade into strips $\left(10 \times 2 \mathrm{~mm}^{2}\right)$ before tests. The dynamic tension tests were performed in multi frequency mode at $0.1 \%$ strain, $1 \mathrm{~Hz}, 25{ }^{\circ} \mathrm{C}$, with a preload of $0.01 \mathrm{~N}$. The static tensile tests were conducted in strain rate mode at a ramp rate of $0.5 \% \mathrm{~min}^{-1}$ with a preload $0.01 \mathrm{~N}$. The mechanical properties for each sample are based on the average value of 5-7 specimens.

\section{Results and discussion}

\subsection{Structural and interfacial characteristics of GO-based films}

GO nanosheets in this paper are prepared from natural graphite powder by chemical exfoliation which is the modified Hummers method. ${ }^{21}$ Fig. 1a shows the diagram of fabrication process and the accompanying chemical mechanisms. Pure GO films, GObased nanocomposite films (noted as GO/CS) were fabricated 


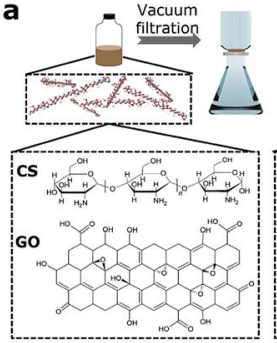

b

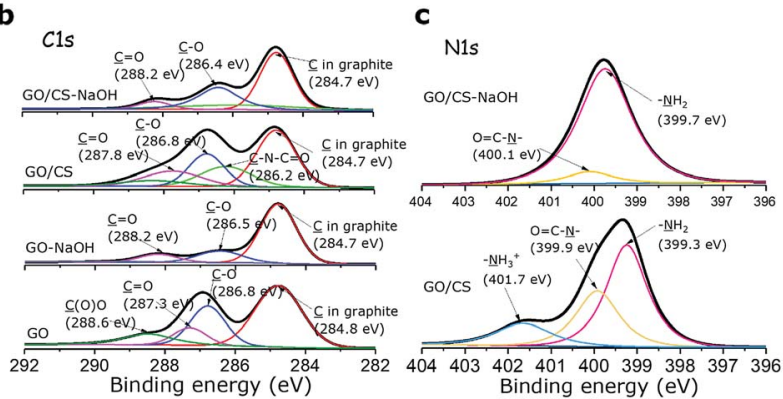

Fig. 1 (a) Schematic illustration of the GO/CS (68/32) and GO/CS (68/ 32) $-\mathrm{NaOH}$ film fabrication process and proposed interlayer interaction mechanism. (b) XPS C 1s spectrum of GO/CS (68/32), GO/CS (68/32)$\mathrm{NaOH}, \mathrm{GO}$ and $\mathrm{GO}-\mathrm{NaOH}$, respectively. (c) $\mathrm{N}$ 1s spectrum of $\mathrm{GO} / \mathrm{CS}$ (68/32) and GO/CS (68/32)- $\mathrm{NaOH}$ films.

by the vacuum assisted filtration method and further treated with alkaline solution (noted as GO/CS-NaOH). Expectedly, the amine groups in CS polymer chains would react with carboxyl groups attached onto GO nanosheets to form amide groups. ${ }^{19,20,22}$ XPS is conducted to characterize the variation of functional groups in GO and GO/CS films before and after alkali treatment as shown in Fig. 1b, c and Table S1. $\dagger$ Interestingly, we find that alkali solution treatment could partially reduce the oxygen-containing groups in $\mathrm{GO}$ sheets, ${ }^{23}$ in which the $\mathrm{C}(\mathrm{O}) \mathrm{O}$ peak vanishes and the $\mathrm{C}-\mathrm{O}$ peak decreases from $25.5 \%$ to $17.2 \%$. Meantime, the $\mathrm{C}=\mathrm{C}$ peaks in graphite ( $\mathrm{C}$ atoms in graphite, $284.7 \mathrm{eV}$ ) increase from $52.7 \%$ to $72.6 \%$, indicating the increasing graphitic domains. As for the GO/CS nanocomposite films, the $\mathrm{C} 1 \mathrm{~s}$ spectra appear a new peak at $286.2 \mathrm{eV}$ (18.1\%) assigned for the $\mathrm{C}-\mathrm{N}$ together with the decrease of $\mathrm{C}(\mathrm{O})$ O peak from $11.2 \%(288.6 \mathrm{eV})$ to $6.6 \%(288.3 \mathrm{eV})$, further confirming the formation of amide linkages between - $\mathrm{COOH}$ in $\mathrm{GO}$ and $-\mathrm{NH}_{2}$ in $\mathrm{CS}$ molecules. The $\mathrm{N}$ 1s spectra of GO/CS in Fig. 1c are consistent well with $\mathrm{C}$ 1s spectra of GO/CS, where the amide group is observed..$^{\mathbf{2 4 , 2 5}}$ After alkali solution treatment, the amide group is broken supported by the significant decrease of amide groups (from $30.6 \%$ to $7.9 \%$ ) in $\mathrm{N}$ 1s spectra, while the amine groups greatly increase from $53.6 \%$ to $92.1 \%$. The presence of amine groups deriving from amide bonds and neutralized amine groups of CS molecules would facilitate the formation of hydrogen bonding networks with hydroxyl groups on GO surface in $\mathrm{GO} / \mathrm{CS}-\mathrm{NaOH}$ sample. Based on the above discussion, we infer that the interlayer bonding types in the GO/CS composite are dominated by both the amide covalent bonding and hydrogen bonding; after alkali treatment, the hydrogen bonding is expected to act as a dominating role at the interface. Additionally, the alkali treatment is favorable for densifying the structure, given the fact that the interlayer spacing in GO/CS$\mathrm{NaOH}$ is less than that of the untreated one (supported by XRD results shown in Fig. $2 b$ ). Such variations in the interfacial bonding types and interlayer spacing are expected to exert influence on mechanical properties of GO/CS nanocomposite. Besides, to clarify the different reduction mechanisms between alkali treatment and HI reduction methods, we also conducted the electric conductivity measurements for alkali treated GObased film and hydroiodic (HI) reduced GO film. The alkali treated GO films show lower conductivity $\left(5.9 \mathrm{~S} \mathrm{~m}^{-1}\right)$ than $\mathrm{HI}$ reduced ones ( $2683.3 \mathrm{~S} \mathrm{~m}^{-1}$ ), indicating that the $\mathrm{GO}$ nanosheets are partially reduced by alkali solution.

With the help of vacuum-assisted filtration method, a systematic series of GO/CS nanocomposite films (GO/CS 100/0, GO/CS 93/ 7, GO/CS 78/22, GO/CS 68/32) were prepared with GO content ranging from 68 to $100 \mathrm{wt} \%$. The exact GO/CS weight ratios were determined by TGA as shown in Fig. S1. $\uparrow$ We first investigate how the intercalated CS polymer affect the microstructure of GO films. Expectedly, the introduction of CS into interlayer gallery would tune the orientation and waviness of individual GO nanosheets. The fracture surface of pure GO film in Fig. 2a reveals that most GO sheets are horizontally aligned with interlayer spacing $0.77 \mathrm{~nm}$ and full width at half-maximum (FWHM) $2.2^{\circ}$ as derived from XRD results in Fig. $2 \mathrm{~b}$, which is consistent with the results in literatures. $^{26,27}$ The obvious layered structure of GO nanosheets could still maintain even that $c a .32 \mathrm{wt} \% \mathrm{CS}$ were intercalated into GO gallery spacing, accompanying the apparent increase in interlayer spacing up to $1.13 \mathrm{~nm}$ and FWHM to $4.60^{\circ}$ even though the peak intensity decays distinctly. After the alkali treatment of GO films, the peak at $11.48^{\circ}$ disappears and the interlayer spacing decreases
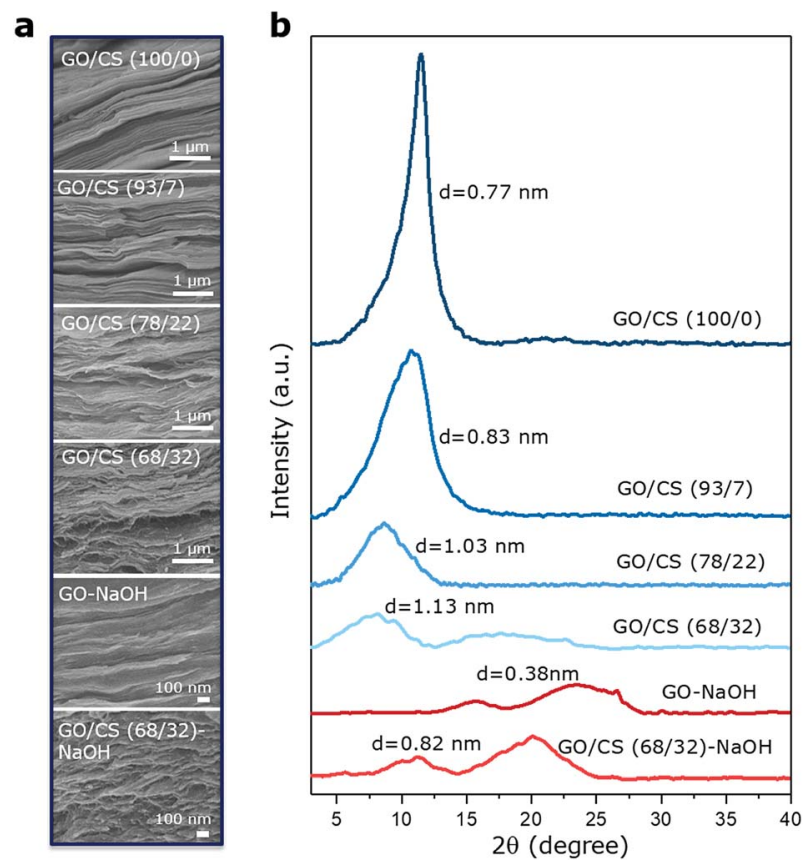

Fig. 2 (a) Side-view morphology in SEM of the curved structures of $\mathrm{GO} / \mathrm{CS}$ nanocomposite films with different weight ratios (100/0, 93/7, $78 / 22,68 / 32$ ) and the fracture surface of the alkali treated $G O$ and $G O /$ CS (68/32) films. (b) XRD patterns of GO/CS films with different weight ratio and alkali treated GO and GO/CS (68/32) films. 
down to $0.38 \mathrm{~nm}$ due to the reduction of the carboxylic and epoxy groups. ${ }^{23}$ Similarly, alkali treated GO/CS (68/32)-NaOH films show narrower interlayer spacing down to $0.82 \mathrm{~nm}$ from $1.13 \mathrm{~nm}$ compared with untreated ones, indicating that alkali penetrates into the dense structure of the GO/CS films. The shoulder peak at $20.08^{\circ}$ might be the crystalline diffraction peaks of CS under the influence of alkaline solution. ${ }^{28}$

\subsection{Static and dynamic mechanical behaviors of GO-based composite films}

Like the previously reported nacre-like based GO composite films, ${ }^{19,29-33}$ the apparent mechanical enhancements are expected for GO/CS composites after intercalation of a given amount of CS inside GO gallery. Here in, benefiting from the synergistic effect of stiff GO sheets, the ductile CS molecules and its dual interfacial crosslinking modes (namely H-bonding and covalent bond), the GO/CS nanocomposite films (93/7, 78/22, 68/32) exhibit higher strength, modulus, toughness than pure GO films as summarized in Fig. 3a and b. For instance, the GO/CS (93/7) composites exhibit $15.5 \pm 1.1 \mathrm{GPa}$, the $63.5 \%$ enhancement of Young's modulus compared to neat GO films. Besides, the GO/CS (68/32)-based nanocomposites exhibit the highest toughness (2.8 $\left.\pm 0.3 \mathrm{MJ} \mathrm{m}^{-3}\right)$ without trading-off strength $(170.7 \pm 15.2 \mathrm{MPa})$
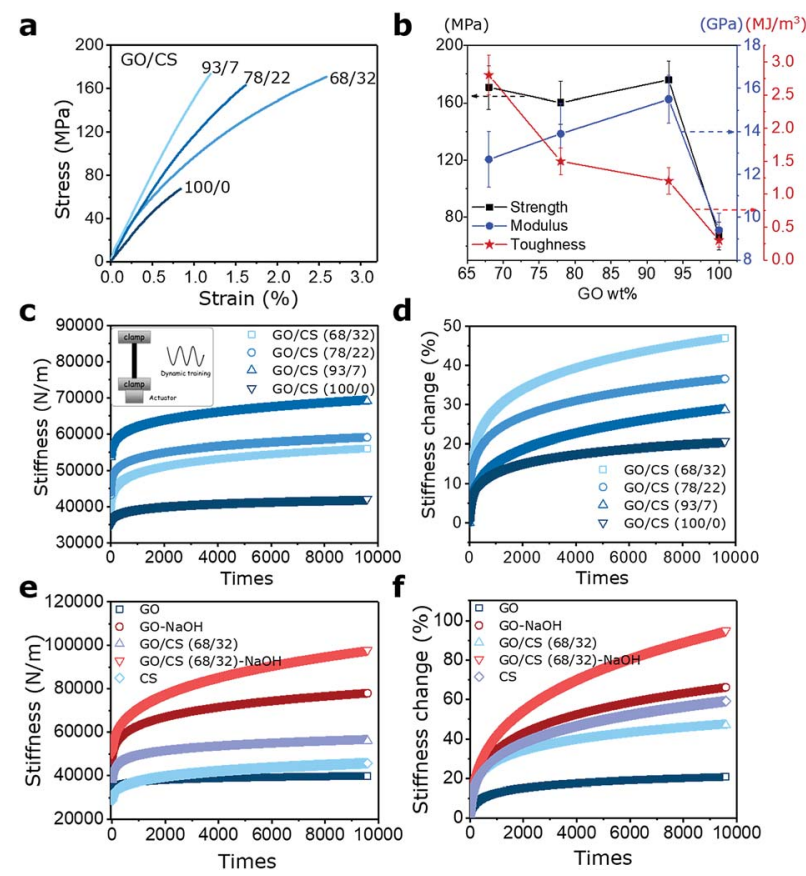

Fig. 3 Static and dynamic mechanical properties of GO-based nanocomposite films. (a) Strain-stress curves of GO/CS (68/32), GO/ CS (78/22), GO/CS (93/7), and GO/CS (100/0). (b) Plots of variation tendency of strength, Young's modulus and toughness of GO/CS nanocomposite films versus varying GO weight ratio, respectively. (c) The GO/CS nanocomposite specimens with different weight ratios $(100 / 0,93 / 7,78 / 22,68 / 32)$ display increasing stiffness versus dynamic tension times when cyclically stretched at $0.1 \%$ amplitude, $1 \mathrm{~Hz}, 0.01 \mathrm{~N}$ preload, $25{ }^{\circ} \mathrm{C}$ using DMA Q800 (inset). (d) Change in stiffness (\%) versus oscillation times during dynamic tests of GO/CS nanocomposites. (e) Stiffness variation and ( $f$ ) change of stiffness versus times of GO, GO-NaOH, GO/CS (68/32), GO/CS (68/32)- $\mathrm{NaOH}$ and CS, respectively. among four specimens. The main deformation mechanisms involved in GO/CS nanocomposite films were related to the slippage of neighbouring GO sheets, stretch and orientation of both GO fillers and CS chains. Further dynamic mechanical tests indicate that pure GO films exhibit $20 \%$ change in stiffness caused by the straightening and reorientation of GO sheets. After incorporating CS molecules inside gallery space in GO sheets, the discernable stiffening behaviors are gradually strengthened in response to the repetitive dynamic tension. Comparatively, the GO/CS (68/32) nanocomposites display 47\% change in stiffness under dynamic tension as shown in Fig. $3 c$ and d, which is more than twofold of that in pure GO films. The apparent enhancement in stiffening role could be attributed to the increased curved features of GO sheets as well as the aligned polymer chains along the loading direction as shown in Fig. 3e and f. CS molecules show apparent self-stiffening role during dynamic tension due to the polymer chain movements. Additionally, unlike previous reports where obvious dimensional changes occur after dynamic loading, ${ }^{11,15}$ such changes in our GO/CS films are quite negligible because of the low-amplitude tensile strain $(\approx 0.1 \%)$ as shown in Fig. S2. $\dagger$ Interestingly, the sample length reduces slightly after dynamic tension, especially for GO/CS films with $22 \%$ and $32 \%$ CS content, which might be due to the resilience of stretched CS chains in the process of oscillation. ${ }^{34,35}$

\subsection{Effects of interlayer interaction on the stiffening performance}

Apart from the layered microstructures, the interlayer movements that are determined by intercalated polymer phase and the interlayer bonding types, can be another important factor in tuning mechanically stiffening behavior of GO nanocomposite films under dynamic load. ${ }^{36,37}$ As discussed above, among four GO/CS 100/0, 93/7, 78/22 and 68/32 nanocomposite specimens, the GO/CS 68/32 composites exhibit the most advantageous mechanical performances in terms of strength and toughness as well as mechanically driven self-stiffening behavior. Thus, the GO/CS 68/32 nanocomposites were employed to investigate the effect of interlayer bonding types on self-stiffening behavior under dynamically mechanical stimuli. To clarify the mechanical responses of alkali treated GO/CS and GO films under dynamic loads, the stiffening behaviors of films as a function of times are monitored as presented in Fig. 3e and f. As expected, all the films displayed monotonously stiffening behaviors over the mechanical training time, and the more prominent stiffening behaviors were observed in samples after alkali treatment. Specially, the GO/CS (68/32)-NaOH film exhibits the highest stiffening behavior among the four different films, change in stiffness being up to $95 \%$ from initial stiffness $50288 \mathrm{~N} \mathrm{~m}^{-1}$ to $97976 \mathrm{~N} \mathrm{~m}^{-1}$. Similarly, the GO-NaOH film also shows higher stiffness increase $\left(78014 \mathrm{~N} \mathrm{~m}^{-1}\right)$ compared to that of GO film (39865 $\left.\mathrm{N} \mathrm{m}^{-1}\right)$.

\subsection{Mechanism of mechanically responsive behavior in GO- based films}

The self-stiffening behaviour of polymer is acknowledged for chain movements (e.g., straightening and reorientation), in 
which the randomly oriented and highly curved chains are supposed to straighten and align in response to dynamic load at a large strain level. ${ }^{38,39}$ In our recent work, we have also demonstrated the mechanically stiffening behaviors of GO films resulted from the stretch of highly curved and misaligned GO nanosheets as well as alignment along the loading direction at a low strain level. Therefore, the larger waviness observed in the cross section of GO/CS (68/32) (Fig. 2a) both with and without treatment could be responsible for its high toughness and stiffening effect.

In order to characterize the microstructural evolutions of $\mathrm{GO} /$ CS films during external dynamic tension and hence confirm its contribution to the self-stiffening behavior, polarized Raman technique is utilized to record spectra of untreated and alkali treated GO/CS films before and after dynamic oscillation. . $^{10,38,40}$ The cross-sectional view of the GO/CS films before and after dynamically stressed is illuminated by an incident laser beam perpendicularly. As shown in Fig. $4 \mathrm{a}$ and $\mathrm{b}$, the angle $(\theta)$ between the base plane of the $\mathrm{GO} / \mathrm{CS}(68 / 32)-\mathrm{NaOH}$ film and the electric field vector of incident laser is altered from $0^{\circ}$ to $360^{\circ}$ with a step of $20^{\circ}$. The angle $(\alpha)$ is the alignment between loading direction and average $\mathrm{GO} / \mathrm{CS}$ (68/32)-NaOH nanosheets as shown in Fig. 4c. The polarized Raman spectra with varying angle $(\theta)$ is recorded and G band intensity $\left(I_{\mathrm{G}}\right)$ is theoretically analyzed. It is observed that $I_{\mathrm{G}}$ reaches a maximum value $I_{\mathrm{G}}(\|)$ when the electric field vector is parallel to the base plane of the GO/CS $(68 / 32)-\mathrm{NaOH}$ film $\left(\theta=0^{\circ}\right.$ and $\left.180^{\circ}\right)$, while arrives at a minimum value $I_{\mathrm{G}}(\perp)$ when perpendicular to the base plane $\left(\theta=90^{\circ}\right.$ and $\left.270^{\circ}\right)$, as shown in Fig. S3.† Specifically, it is reported that the $I_{\mathrm{G}}(\|) / I_{\mathrm{G}}(\perp)$ is a

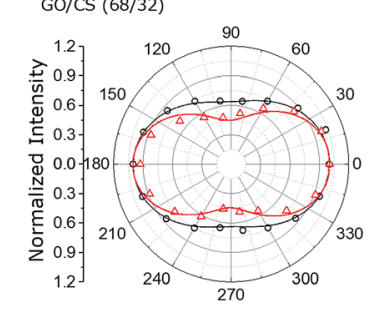

c

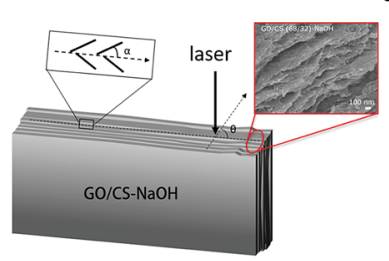

b

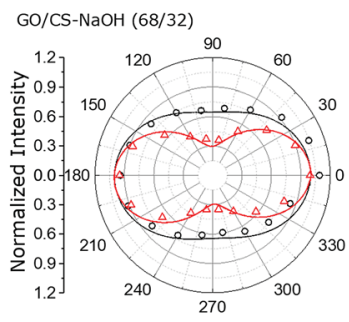

d

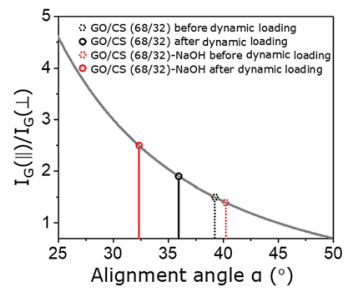

Fig. 4 (a) The G band intensity $\left(I_{G}(\theta)\right)$ as a function of angle $(\theta)$ between the base plane of samples and the electric field vector of illuminated laser for (a) GO/CS (68/32) films and (b) alkali treated GO/CS (68/32) films (black circles are for samples before dynamic loading, red triangles are after dynamic loading). (c) Schematic illustration of the measurement methodology of Raman analysis for dynamically unstressed and stressed GO/CS-NaOH films. $\alpha$ is the alignment angle between loading direction and average $\mathrm{GO} / \mathrm{CS}-\mathrm{NaOH}$ nanosheets. $\theta$ is the angle between the base plane of the GO/CS (68/32)- $\mathrm{NaOH}$ and the electric field vector of incident laser beam. Inset is the side-view SEM image of alkali treated GO/CS (68/32) film after 10000 cycles dynamic treatment. (d) $I_{\mathrm{G}}(\|) / I_{\mathrm{G}}(\perp)$ ratio as a function of alignment angle $(\alpha)$ of nanosheets. equal to $\cot ^{2} \alpha$, which indicates that $\alpha$ decreases with an increasing $I_{\mathrm{G}}(\|) / I_{\mathrm{G}}(\perp)$ ratio. Moreover, for alkali treated $\mathrm{GO} / \mathrm{CS}$ $(68 / 32)$ sample, the $I_{\mathrm{G}}(\|) / I_{\mathrm{G}}(\perp)$ is significantly increased up to 2.5 compared with the unstressed one at the ratio of 1.4, indicating the alignment improvement of GO nanosheets along loading direction. Meantime, the alignment angles of GO nanosheets before dynamic loading averagely distribute at $40^{\circ}$ in respect to loading direction as shown in Fig. 4d. After dynamic loading the distributed angle decreases to $32^{\circ}$, indicating that the occurring of microstructural evolution which improves the nanosheet alignments over the dynamic loading. Compared with the untreated $\mathrm{GO} / \mathrm{CS}$ films (the alignment angle is altered from averagely $39^{\circ}$ to $36^{\circ}$ ), alkali treated GO/CS films exhibit more significant microstructure evolution and increase in alignment. A mechanically stiffer sample is hence achieved as a result of improved alignment of the laminated structures (Fig. 5).

Furthermore, from the perspective of interfacial bonding, $\mathrm{H}$ bonding interaction has been proved to be beneficial to facilitate stiffening performance of materials under dynamic load because $\mathrm{H}$-bond can easily break and reform under external load due to the rotational degrees of freedom. ${ }^{10}$ Herein, the presence of amide covalent bonding at interface in $\mathrm{GO} / \mathrm{CS}$ can significantly improve bulk mechanical properties of composites, but its irreversibility feature is expected to weaken stiffening performance. ${ }^{10,34}$ Instead, with the aid of alkali treatment, the amide bonds at interface in $\mathrm{GO} / \mathrm{CS}$ composites were transformed into $\mathrm{H}$ bonding instead, which would benefit both the movements of GO nanosheets and CS molecules at the interface.

\subsection{Improving the static mechanical properties by dynamic tension}

The presence of polymer phase and variation in interfacial interaction types successfully enhance the dynamically responsive behavior, which may provide an effective way to strengthen the static mechanical properties of bulk materials. After the dynamical oscillation processes in the GO based films, we further conduct the static tensile test to verify the mechanical enhancement in both GO and GO/CS (68/32) composite films. As shown in Fig. 5a, the static mechanical properties of stressed GO/CS (68/32)-NaOH film could reach up to $35.1 \pm$ 4.2 GPa in modulus, $614.0 \pm 36.6 \mathrm{MPa}$ in strength and $9.3 \pm 1.7$ $\mathrm{MJ} \mathrm{m}{ }^{-3}$ in toughness, in comparison with the $\mathrm{GO} / \mathrm{CS}$ film (12.7 $\pm 1.3 \mathrm{GPa}$ in modulus, $170.7 \pm 15.2 \mathrm{MPa}$ in strength and $2.8 \pm$ $0.3 \mathrm{MJ} \mathrm{m}^{-3}$ in toughness). Compared with other GO-based films (enhanced via divalent and trivalent ions; polymers that can form covalent bonding, hydrogen bonding, $\pi-\pi$ conjugated interactions; reduced GO based with synergistic interactions, etc.), ${ }^{27,29,31,34,41-46}$ the GO/CS-NaOH stressed film show advantageous modulus and strength. Fig. 5b summarizes the mechanical properties of GO-based films in our work and other GO-based nanocomposites reported in literatures (detailed information see Table S2 $\dagger$ ). These observations point out that besides chemical treatments, the GO-based materials can be reinforced by a simple mechanical oscillation because of their mechanically responsive behaviors. An experimental routine is demonstrated in Fig. 5b: mechanical properties of GO film can 

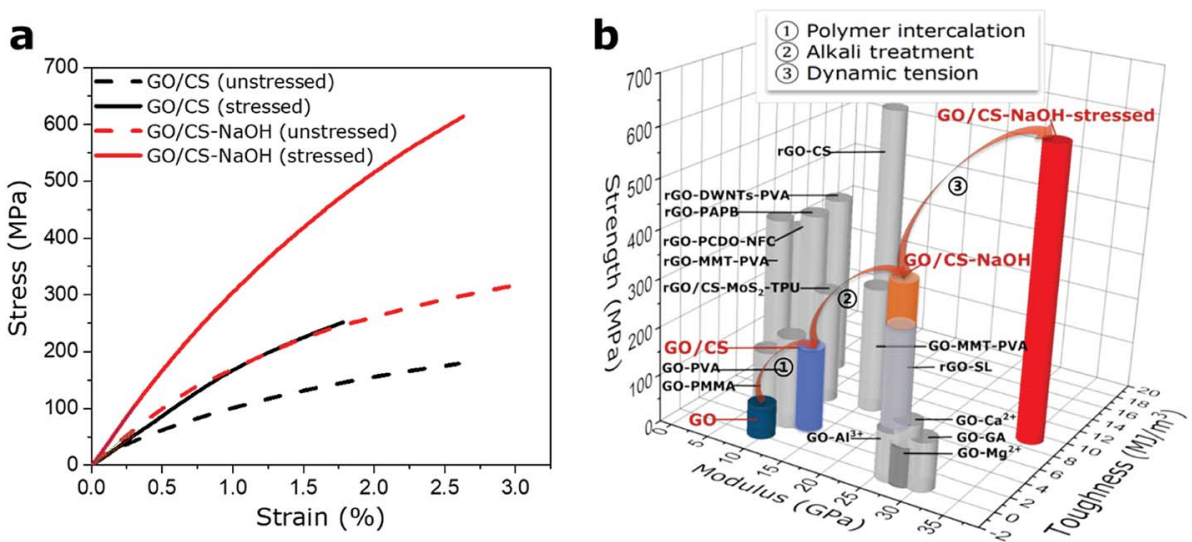

Fig. 5 (a) Strain-stress curves of GO and GO/CS (68/32)- NaOH nanocomposite films before and after dynamic tension. (b) Strength, modulus and toughness of other GO-based nanocomposites (grey bars) compared with that of our works (colorful bars). Mechanical properties of GO film have been enhanced step by step via compositing with polymer, modifying interlayer interaction, and being stiffened under external dynamic loading.

be enhanced via polymer intercalation, interface alteration, and finally mechanical stimuli step by step. Additionally, the difference in terms of Young's modulus, tensile strength and toughness of various GO based composite films both in this work and literature might relate to the following issues: the mechanical properties of individual GO sheets, interface adhesion between reinforcing fillers and intercalated polymer, polymer volume fractions and its morphology at interface, and microstructural features of composite films including nanofiller alignment, waviness, sheet sizes.

\section{Conclusions}

Inspired by the admirable biological tissues which are adaptive to external stimuli, we report a mechanically stiffening GO-based nanocomposite films in response to dynamic tension with as high as $95 \%$ increase in stiffness, meanwhile the static mechanical properties reach $35.1 \pm 4.2 \mathrm{GPa}$ in modulus, $614.0 \pm$ $36.6 \mathrm{MPa}$ in strength and $9.3 \pm 1.7 \mathrm{MJ} \mathrm{m}^{-3}$ in toughness. The stiffening performance of GO-based films can be tuned through altering layered structure by intercalating different contents of polymer, and tailoring interlayer adhesions between polymer and GO nanosheets via alkali treating method. After dynamic loading, the mechanically responsive GO based nanocomposite films can be stiffened and achieve excellent strength and Young's modulus. Alternatively, our results will be beneficial to understand the mechanically responsive mechanism of the special structural materials and exploit the stiffening behavior to pursue higher performance nanocomposites before practical application.

\section{Conflicts of interest}

There are no conflicts to declare.

\section{Acknowledgements}

This project was supported by the National Natural Science Foundation of China (Grant No. 21474023), National Key Basic Research Program of China (Grant No. 2013CB934203).

\section{References}

1 L. Montero de Espinosa, W. Meesorn, D. Moatsou and C. Weder, Chem. Rev., 2017, 117, 12851-12892.

2 J. R. Capadona, K. Shanmuganathan, D. J. Tyler, S. J. Rowan and C. Weder, Science, 2008, 319, 1370-1374.

3 T. Motokawa, Comp. Biochem. Physiol., C: Comp. Pharmacol., 1981, 70, 41-48.

4 A. Chamay and P. Tschantz, J. Biomech., 1972, 5, 173-180.

5 G. E. Fantner, J. Adams, P. Turner, P. J. Thurner, L. W. Fisher and P. K. Hansma, Nano Lett., 2007, 7, 2491-2498.

6 R. De, A. Zemel and S. A. Safran, Nat. Phys., 2007, 3, 655-659.

7 K. M. Schmoller, P. Fernandez, R. C. Arevalo, D. L. Blair and A. R. Bausch, Nat. Commun., 2010, 1, 134-141.

8 L. Mullins, Rubber Chem. Technol., 1969, 42, 339-362.

9 L. Cao, Y. Wang, P. Dong, S. Vinod, J. T. Tijerina, P. M. Ajayan, Z. Xu and J. Lou, Small, 2016, 12, 3723-3731.

10 Z. Dai, Y. Wang, L. Liu, X. Liu, P. Tan, Z. Xu, J. Kuang, Q. Liu, J. Lou and Z. Zhang, Adv. Funct. Mater., 2016, 26, 7003-7010.

11 B. J. Carey, P. K. Patra, L. Ci, G. G. Silva and P. M. Ajayan, ACS Nano, 2011, 5, 2715-2722.

12 P. S. Owuor, C. S. Tiwary, R. Koizumi, M. Soto, A. C. Hart, E. V. Barrera, R. Vajtai, J. Lou and P. M. Ajayan, Adv. Eng. Mater., 2017, 19, 1600756.

13 Y. Li, P. Zhou, F. An, Y. Liu and C. Lu, ACS Appl. Mater. Interfaces, 2017, 9, 5653-5659.

14 E. Senses and P. Akcora, Macromolecules, 2013, 46, 18681874.

15 A. Agrawal, A. C. Chipara, Y. Shamoo, P. K. Patra, B. J. Carey, P. M. Ajayan, W. G. Chapman and R. Verduzco, Nat. Commun., 2013, 4, 1739-1745.

16 W. Gao, L. B. Alemany, L. Ci and P. M. Ajayan, Nat. Chem., 2009, 1, 403-408.

17 Y. Zhu, S. Murali, W. Cai, X. Li, J. W. Suk, J. R. Potts and R. S. Ruoff, Adv. Mater., 2010, 22, 3906-3924.

18 M. Rinaudo, Prog. Polym. Sci., 2006, 31, 603-632.

19 S. Wan, J. Peng, Y. Li, H. Hu, L. Jiang and Q. Cheng, ACS Nano, 2015, 9, 9830-9836. 
20 M. Fang, J. Long, W. Zhao, L. Wang and G. Chen, Langmuir, 2010, 26, 16771-16774.

21 W. S. Hummers Jr and R. E. Offeman, J. Am. Chem. Soc., 1958, 80, 1339.

22 W. Hung, S. Chang, R. G. Lecaros, Y. Ji, Q. F. An, C. Hu, K. Lee and J. Lai, Carbon, 2017, 117, 112-119.

23 X. Fan, W. Peng, Y. Li, X. Li, S. Wang, G. Zhang and F. Zhang, Adv. Mater., 2008, 20, 4490-4493.

24 G. Lawrie, I. Keen, B. Drew, A. Chandler-Temple, L. Rintoul, P. Fredericks and L. Grøndahl, Biomacromolecules, 2007, 8, 2533-2541.

25 I. Amaral, P. Granja and M. Barbosa, J. Biomater. Sci., Polym. Ed., 2005, 16, 1575-1593.

26 D. A. Dikin, S. Stankovich, E. J. Zimney, R. D. Piner, G. H. Dommett, G. Evmenenko, S. T. Nguyen and R. S. Ruoff, Nature, 2007, 448, 457-460.

27 S. Park, K.-S. Lee, G. Bozoklu, W. Cai, S. T. Nguyen and R. S. Ruoff, ACS Nano, 2008, 2, 572-578.

28 Y. Yang, X. Wang, F. Yang, H. Shen and D. Wu, Adv. Mater., 2016, 28, 7178-7184.

29 Y. Zhang, S. Gong, Q. Zhang, P. Ming, S. Wan, J. Peng, L. Jiang and Q. Cheng, Chem. Soc. Rev., 2016, 45, 2378-2395.

30 H. B. Yao, J. Ge, L. B. Mao, Y. X. Yan and S. H. Yu, Adv. Mater., 2014, 26, 163-187.

31 J. Duan, S. Gong, Y. Gao, X. Xie, L. Jiang and Q. Cheng, ACS Appl. Mater. Interfaces, 2016, 8, 10545-10550.

32 J. Zhu, H. Zhang and N. A. Kotov, ACS Nano, 2013, 7, 48184829.
33 K. W. Putz, O. C. Compton, M. J. Palmeri, S. T. Nguyen and L. C. Brinson, Adv. Funct. Mater., 2010, 20, 3322-3329.

34 Y. Gao, L.-Q. Liu, S.-Z. Zu, K. Peng, D. Zhou, B.-H. Han and Z. Zhang, ACS Nano, 2011, 5, 2134-2141.

35 L. Liu, Y. Gao, Q. Liu, J. Kuang, D. Zhou, S. Ju, B. Han and Z. Zhang, Small, 2013, 9, 2466-2472.

36 Z. Dai, G. Wang, L. Liu, Y. Hou, Y. Wei and Z. Zhang, Compos. Sci. Technol., 2016, 136, 1-9.

37 G. Wang, E. Gao, Z. Dai, L. Liu, Z. Xu and Z. Zhang, Compos. Sci. Technol., 2017, 149, 220-227.

38 Z. Dai, Y. Gao, L. Liu, P. Pötschke, J. Yang and Z. Zhang, Polymer, 2013, 54, 3723-3729.

39 R. Haward, Macromolecules, 1993, 26, 5860-5869.

40 Q. Liang, X. Yao, W. Wang, Y. Liu and C. P. Wong, ACS Nano, 2011, 5, 2392-2401.

41 C. N. Yeh, K. Raidongia, J. Shao, Q. H. Yang and J. Huang, Nat. Chem., 2014, 7, 166-170.

42 K. W. Putz, O. C. Compton, M. J. Palmeri, S. T. Nguyen and L. C. Brinson, Adv. Funct. Mater., 2010, 20, 3322-3329.

43 Y. Q. Li, T. Yu, T. Y. Yang, L. X. Zheng and K. Liao, Adv. Mater., 2012, 24, 3426-3431.

44 Y. Tian, Y. Cao, Y. Wang, W. Yang and J. Feng, Adv. Mater., 2013, 25, 2980-2983.

45 S. Wan, Y. Li, J. Peng, H. Hu, Q. Cheng and L. Jiang, ACS Nano, 2015, 9, 708-714.

46 K. Hu, L. S. Tolentino, D. D. Kulkarni, C. Ye, S. Kumar and V. V. Tsukruk, Angew. Chem., Int. Ed., 2013, 52, 13784-13788. 